\title{
Método Adaptativo de Programação da Produção Apoiado por um Sistema de Medição de Desempenho e Melhoria Contínua
}

\author{
Helder Carlo Belan ${ }^{1}$, Jandira Guenka Palma ${ }^{1}$ \\ ${ }^{1}$ Departamento de Computação - Universidade Estadual de Londrina (UEL) \\ Caixa Postal 6001 - 86051-990 - Londrina - PR - Brazil \\ hcbelanegmail.com, jgpalma@uel.br
}

\begin{abstract}
This paper proposes a production scheduling method that adapts to the scenario production of each industry. Named as MAP-Prod this method should incorporate characteristics of reactive and proactive approaches. Thus, the cycle of adaptation is characterized by improvement actions found through the gathering information and analysis of indicators in a performance measurement system. These actions are accompanied by a continuous improvement system to diagnose their effectiveness. A simulation is then performed with real data from an industry in order to verify the results that can be obtained with the method.
\end{abstract}

Resumo. Este trabalho propõe um método de programação da produção que se adapte ao cenário produtivo de cada indústria. Denominado de MAP-Prod o método deve incorporar características das abordagens reativa e proativa, onde o ciclo de adaptação será caracterizado por ações de melhoria encontradas através da coleta de informações e análise de indicadores em um sistema de medição de desempenho. Estas ações serão acompanhadas por um sistema de melhoria contínua para diagnosticar a eficácia das mesmas. Uma simulação então é realizada com dados reais de uma indústria para averiguar os resultados que podem ser obtidos com o método.

\section{Introdução}

A atividade de Planejamento, Programação e Controle da Produção (PPCP), na manufatura, desempenha um papel de extrema importância no ambiente de alta competição em que as empresas se encontram. Especificamente no sequenciamento de operações, atividade diretamente associada ao PPCP, no qual as decisões que direcionam a ordem em que os produtos devem ser fabricados, respeitando prioridades e restrições impostas pelo processo, impactam consideravelmente nos prazos de entrega do produto para o cliente e nos custos.

Apesar de ser considerada uma atividade complexa pela quantidade de variáveis que devem ser consideradas, existem vários métodos e técnicas para auxiliar a programação da produção, inúmeros algoritmos conseguem em tempo hábil encontrar boas soluções para cada tipo de processo produtivo. Entretanto há um grande problema em ambientes reais, onde nem sempre a programação escolhida pode ser seguida à risca, pois eventos inesperados podem gerar discrepâncias entre o que foi planejado e o que realmente aconteceu. 
Existe uma dinamicidade do ambiente, onde alterações como mudanças de funcionários, troca de ferramentas, inserção de novos produtos e mau funcionamento de máquinas podem gerar uma necessidade de ajuste dos parâmetros e técnicas de programação utilizados; portanto um método adaptativo que se adeque a essas mudanças pode melhorar a programação com os novos parâmetros mais próximos do real.

Pode-se então equiparar essas adaptações do modelo da programação da produção a um processo de melhoria contínua onde objetiva-se encontrar os melhores parâmetros e as melhores técnicas de sequenciamento de atividades para a atual configuração do processo produtivo da empresa.

A ideia de melhoria contínua está relacionada à capacidade de resolução de problemas por meio de pequenos passos, alta frequência e ciclos curtos de mudança. Esses ciclos de mudança são causados pela alternância de momentos de ruptura e de controle no desempenho [Bessant et al. 1994, Attadia and Martins 2003]. Para garantir que o processo de melhoria alcance seus objetivos tem-se a medição de desempenho como arma fundamental para mensurar os avanços em cada adaptação e orientar corretamente as ações para convergência de um método de programação condizente com as realidades do ambiente produtivo da empresa.

Neste trabalho é proposto um método de programação da produção que aja de maneira adaptativa, ajustando os parâmetros e técnicas utilizadas para o sequenciamento de atividades de acordo com as orientações de um sistema de melhoria contínua e medição de desempenho.

Na continuação desta seção serão descritos algumas definições e trabalhos semelhantes; já na seção 2 será exemplificado o método proposto com a arquitetura entre os sistemas. Por último, na seção 3 serão mostrados os resultados obtidos com a aplicação do método proposto em uma indústria do setor moveleiro.

\subsection{Programando a produção em um ambiente cheio de incertezas}

Em vários segmentos da indústria a programação da produção é uma tarefa altamente complexa que envolve inúmeras variáveis quanto à disponibilidade e capacidade de recursos, disponibilidade de materiais e principalmente restrições quanto ao processo de fabricação, com isso o uso de ferramentas avançadas de programação (APS - $A d$ vanced Planning System) é quase obrigatório para quem quer alcançar bons resultados na otimização dos recursos fabris.

Devidamente configuradas as ferramentas APS utilizam algoritmos computacionais que de forma matemática ou heurística conseguem encontrar boas soluções para o sequenciamento das atividades no chão de fábrica. Este sequenciamento gerado durante a fase de planejamento é chamado de programação preditiva, pois é o que se espera ocorrer caso os parâmetros utilizados estejam em conformidade com o ambiente fabril. Porém é fato que durante a execução do planejamento a empresa ainda está sujeita as ocorrências inesperadas e imprevisíveis que podem impactar consideravelmente a programação como era prevista. Esses problemas se devem a característica dinâmica do ambiente fabril e dificilmente consegue-se evitá-los.

Dentre as causas que geram essas rupturas no planejamento podemos citar: atividades que duram mais ou menos tempo que o previsto; recursos podem ficar in- 
disponíveis; o fornecedor pode atrasar a entrega da matéria-prima; prazos de entrega podem mudar de acordo com a pressão de clientes ou estratégias da gerência; retrabalhos podem ser incorporados para arrumar falhas no produto; dependendo do segmento até alterações no tempo podem trazer impactos na produção [Herroelen and Leus 2005].

Pensando nisso, vários autores propuseram novos métodos para este ambiente dinâmico, os quais podem ser classificados em três família: abordagem reativa, progressiva e proativa [Aytug et al. 2005, Herroelen and Leus 2005, Bidot et al. 2009]. Na primeira família as decisões podem ser tomadas à medida que os eventos vão ocorrendo no chão de fábrica, ou seja, o sistema deve ser hábil o suficiente para coletar as informações, processá-las e retornar uma nova programação válida em tempo real.

Já a segunda família utiliza-se de uma abordagem progressiva para gerar uma programação preditiva em pequenos espaços de tempo já predefinidos, onde o risco de incertezas é bem menor [Bidot et al. 2009].

Por fim a família de métodos com abordagem proativa assemelha-se ao processo humano de aprendizado e melhoria, os métodos desta classe se utilizam de heurísticas e uma base de conhecimento para prever os eventos que podem ocorrer durante a execução da programação e com isso gerar um sequenciamento preditivo cada vez mais confiável e menos passível de rupturas [Bidot et al. 2009].

\subsection{Métodos Adaptativos}

Pode-se encontrar um bom exemplo de método adaptativo no trabalho de Mehta e Uzsoy (1998) onde foi montado um sistema proativo/reativo utilizando-se da estratégia de inserir tempos ociosos na programação para minimizar os efeitos de paradas de máquinas inesperadas. Com isso os autores conseguiram gerar soluções preditivas próximas ao executado no que se refere ao tempo de lead time ${ }^{1}$ dos produtos.

Os resultados obtidos no trabalho de Mehta e Uzsoy (1998) mostram que as soluções preditivas obtidas com a adição do tempo ocioso ao tempo de processamento das atividades tiveram desempenho semelhante ao trabalho realizado na prática mesmo com a ocorrência de paradas imprevistas.

Trabalho semelhante foi proposto por Lambrechts, Demeulemeester e Herroelen (2008) novamente utilizando a abordagem proativa e a reativa para maximizar a estabilidade das soluções geradas. As estratégias utilizadas dão prioridade maior às operações com maior dependência e recursos com histórico maior de paradas imprevistas, assim essas operações ganham certa proteção quanto a indisponibilidades dos recursos necessários.

O autor ainda prevê uma estratégia reativa utilizando o algoritmo de Busca Tabu para procurar uma nova programação que seja passível de execução e com o menor grau de alteração com a anterior visando a estabilidade do sistema. Os resultados mostrados por Lambrechts, Demeulemeester e Herroelen (2008) alcançam a marca surpreendente de melhoria de $96 \%$ de estabilidade com a utilização destas estratégias.

A característica em comum destes trabalhos e de outros que utilizam a abordagem proativa é que a evolução do modelo de programação depende muito do entendi-

\footnotetext{
${ }^{1}$ Lead time é considerado o tempo de completude de uma tarefa, ou seja, o intervalo entre o início de sua primeira operação até o término da última
} 
mento sobre o processo produtivo e da influência das alterações nos parâmetros inicias da programação na prevenção de rupturas do planejamento, ou seja, este processo muitas vezes é realizado de forma empírica e requerem certo cuidado quanto à extrapolação de prevenções aos riscos e subestimação da capacidade produtiva, fatos estes que motivam e dão sustentação ao uso de sistemas de apoio a gestão do processo como forma de mensurar e acompanhar as modificações feitas no modelo. Os sistemas referidos aqui podem ser chamados de sistema de medição de desempenho e melhoria continua.

\subsection{Ciclo de melhoria e medição de desempenho de sistemas dinâmicos}

Segundo Bessant (1994) a ideia de melhoria contínua está relacionada à capacidade de resolução de problemas por meio de pequenos passos, alta frequência e ciclos curtos de mudança. Em modelos de programação com a abordagem proativa o processo de melhoria contínua será dado através de ciclos de acompanhamento com período igual ao horizonte de planejamento. Em cada fim de ciclo os indicadores serão calculados e as adaptações sugeridas pelo método serão implementadas para a programação do próximo período.

Categorizando as atividades dentro do ciclo PDCA (Plan, Do, Check, Act) temos as escolhas e alterações do modelo de programação visando o aumento de desempenho em gerar soluções preditivas na etapa de planejamento $(\mathrm{P})$. A definição dos novos parâmetros do sequenciamento de operações como tempos, buffers e escolha das técnicas é tratada na etapa de execução (D). Após o horizonte de plenejamento, os resultados são avaliados através dos indicadores de desempenho $(\mathrm{C})$ e podem ser definitivos ou não de acordo com a evolução de desempenho mostrado (A). Então novas ações podem ser propostas e inicia-se outro ciclo.

Para garantir que o processo de melhoria alcance seus objetivos tem-se a medição de desempenho como arma fundamental para mensurar os avanços em cada adaptação e orientar corretamente as ações para convergência de um método de programação condizente com as realidades do ambiente produtivo da empresa. Para Savolainen (1999) a medição de desempenho é uma arma poderosa para alcançar os objetivos de melhoria contínua, e levando em conta o fato de que esse é um processo evolutivo, a medição de desempenho pode dar sustentação para o ciclo de aprendizado de melhoria contínua organizacional [Attadia and Martins 2003].

Os principais indicadores relacionados a programação da produção referem-se ou a qualidade das soluções geradas ou quanto a sua execução. Muitas empresas têm diferentes pontos de vista sobre o desempenho da programação da produção, existem diversos objetivos que podem ser adotados por cada uma, mas geralmente vão estar relacionados à utilização de recursos, prazo de entrega ou custo de produção.

Existem ainda indicadores de desempenho relacionados à relação entre a solução programada e a sua execução, estes indicadores são essenciais para o processo de melhoria contínua do sistema em questão, por isso neste trabalho dá-se um destaque maior aos indicadores de estabilidade e robustez da solução.

A estabilidade só pode ser medida após a execução das atividades, já que a mesma está relacionada ao quão diferente foi a execução do planejamento. Para Bidot et al. (2009) uma programação é dita estável se as ações tomadas durante a execução estão próximas do planejamento no sequenciamento preditivo. A estabilidade de uma solução pode ser medida através da somatória das diferenças entre o início planejado e o início real 
de cada atividade mais a diferença entre a sequência de atividades planejada e a ordem que foi realmente executada [Ouelhadj and Petrovic 2009].

Provavelmente um dos indicadores mais utilizados para sistemas de ambiente dinâmico, a robustez mede o que deve realmente importar para as empresas: a relação entre a qualidade da programação preditiva e a qualidade da execução no chão de fábrica. Billaut et al. (2010) define como uma solução robusta aquela em que sua performance é bastante indiferente às incertezas relacionadas a sua execução.

Por causa das características evolutivas dos métodos de programação da produção com abordagem proativa, a maioria das vantagens que os sistemas de medição de desempenho e melhoria contínua possibilitam são aplicáveis ao problema, como a maior visibilidade do processo, estabelecimento de metas e rastreabilidade entre ações.

\section{MAP-Prod - Um Método Adaptativo de Programação de Produção da Produção com Apoio de um Sistema de Medição de Desempenho e Melhoria Contínua}

Como foi descrito por vários autores o processo de adaptação do sistema de programação preditiva pode utilizar inúmeras estratégias para deixar as soluções cada vez mais estáveis e robustas [Mehta and Uzsoy 1998, Lambrechts et al. 2008]. Com isso os métodos adaptativos minimizam os impactos de eventos inesperados no chão de fábrica e proporcionam uma importância maior à programação da produção já que aumenta a possibilidade de melhorias em seu processo, permitindo que a empresa entre em um processo de melhoria semelhante ao descrito por Merli (1993) .

Assim, este trabalho desenvolveu um método de melhoria contínua da linha de produção, baseado em indicadores de desempenho que geram adaptações constantes no planejamento da produção conforme a dinâmica do ambiente. A proposta foi denominada MAP-Prod (Método Adaptativo de Programação da Produção) e apresentada na Figura 1. O método propõe integração dos sistemas ERP - Enterprise Resources Planning, APS, MES - Manufacturing Execution System e SMD/SMC - Sistema de Medição de Desempenho e Melhoria Contínua com atualizações constantes dos parâmetros empregados no sequenciamento pelo APS a partir dos indicadores colhidos pelo SMD/SMC.

Para auxiliar o gerenciamento deste processo e garantir uma melhor aderência do modelo à realidade da empresa, este trabalho propõe que todo o processo de melhoria do método de programação da produção seja acompanhado por um sistema de medição de desempenho e melhoria contínua com o intuito de mensurar, adaptar as realidades momentâneas (aplicar o método adaptativo) e rastrear as ações de melhoria (adaptações no modelo) que ocorrem durante os ciclos de produção.

O MAP-Prod emprega vários tipos de sistemas, os quais podem ser encontrados no mercado com diversos nomes e siglas, estes sistemas são classificados na literatura de acordo com o conjunto de funcionalidades definidas como padrão para cada categoria. Neste trabalho propõe-se o incremento de algumas funcionalidades que estão em destaque na Figura 1, tal como a identificação e atualização de ordens durante uma ruptura para o sistema MES, a atualização da situação dos recursos em tempo real e atualização dos parâmetros a cada ciclo de melhoria no sistema APS, estas funcionalidades podem ser desenvolvidas internamente no produto ou através de interfaces de integração. 


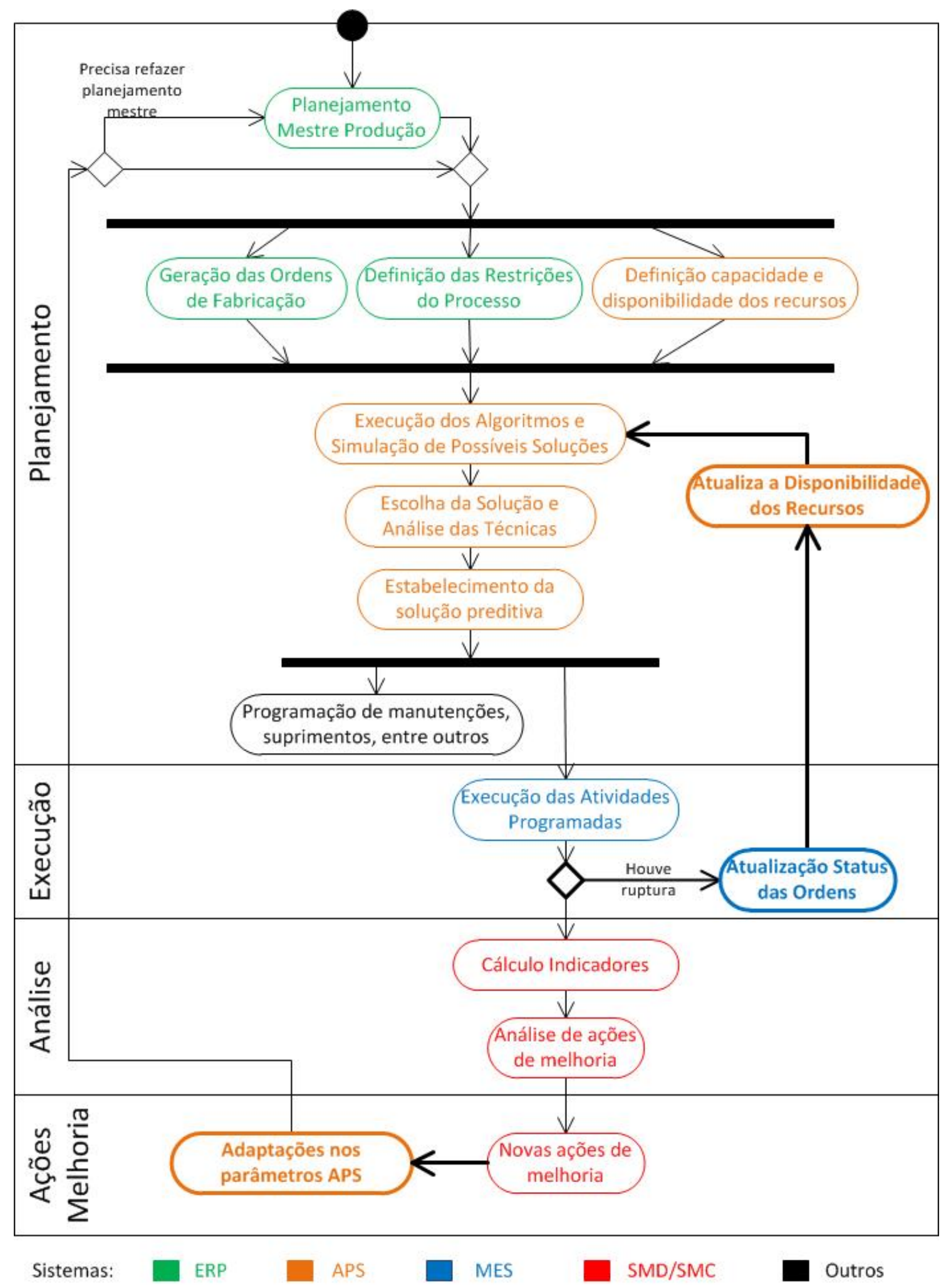

Figure 1. Método MAP-PROD "Método Adaptativo da Programação da Produção" (diagrama de atividade em UML de cada horizonte curto de planejamento) 
O MAP-Prod é dividido em 4 fases: Planejamento, Execução, Análise e Ações de melhoria no texto a seguir foi desdobrado cada uma das fases para melhor entendimento do processo.

\subsection{Fase do Planejamento}

Durante esta fase são realizados todos os níveis de planejamentos e definições dos parâmetros do processo de fabricação. Primeiramente são revistas as definições do Plano Mestre de Produção (PMP) para que seja concretizado parte dele no próximo horizonte curto, as ordens então são geradas atendendo a demanda descrita no PMP.

A parametrização da ferramenta APS deve sempre ser revista informando corretamente a capacidade e disponibilidade dos recursos fabris e caso haja mudanças nos processos as restrições do mesmo também devem ser atualizadas. Estabelecidos todos os parâmetros necessários, o PPCP inicia o processo de simulações utilizando-se da ferramenta APS e os algoritmos disponíveis na mesma. Além do processo computacional que envolve a execução dos algoritmos, a experiência dos usuários pode ajudar com ajustes manuais nas soluções obtidas.

Nesta fase deve-se atentar para o fato que a execução dos algoritmos pode estar ocorrendo para o início de mais um ciclo de produção ou para montar uma programação parcial após a ocorrência de uma ruptura. No primeiro caso tem-se somente a preocupação em gerar a melhor solução para o próximo nível aliada a revisão dos parâmetros da ferramenta, já no segundo caso o tempo dispendido na simulação é outro ponto importante para se preocupar, já que quanto mais rápido a nova solução é gerada, menores são os prejuízos com a ocorrência da ruptura.

Um processo de divulgação deve ser estabelecido para que a solução escolhida na ferramenta APS seja passada para todos os responsáveis na empresa, é nesta fase em que os dados sobre o cronograma de produção é transmitido para os sistemas de gestão integrado (ERP) e o sistema que acompanhará a execução no chão de fábrica (MES). Como um dos propósitos da programação é servir de base para outros planejamentos e como forma de integração entre áreas cooperantes, com isso podem-se ressaltar algumas áreas onde a programação poderia ser útil como a logística e manutenção.

\subsection{Fase da Execução}

O sistema MES (Manufacturing Execution System) é o responsável por gerenciar a execução da programação no chão de fábrica. Ele pode fazer uso de painéis para realizar a liberação das ordens de acordo com a sequência estabelecida além de gerenciar as máquinas e funcionários que estão executando as tarefas.

Em posse da solução preditiva, o MES pode fazer o controle e liberação das ordens de produção para as células de trabalho, supervisionando o trabalho em tempo real. Alertas podem ser gerados após algum atraso significativo ou qualquer outro desvio do planejamento. Dependendo do desvio identificado pelo sistema MES, a solução preditiva pode deixar de ser factível, como a quebra de uma máquina, inserção de novas ordens devido a urgência de retrabalhos e reposições de peças. Por isso o sistema MES deve ter políticas pré-definidas para identificação de rupturas para que de forma automática ou de forma manual pelos operadores PPCP possam iniciar o procedimento reativo da 
programação, ou seja, atualizar o que foi feito até o momento e reprogramar as tarefas pendentes com o novo cenário.

\subsection{Fase de Análise}

Durante a Análise da Execução e Estratégia de Melhoria, ao fim do horizonte curto de planejamento chega-se ao fim de mais um ciclo de melhoria onde os resultados devem ser analisados e novas ações devem ser propostas para os problemas identificados.

O desempenho da produção deverá ser medido de acordo com um conjunto de indicadores estipulados para cada empresa. Esses indicadores devem representar os diversos departamentos da empresa. Para garantir a utilização desses indicadores durante o processo de adaptação da ferramenta de programação é necessário adicionar indicadores de desempenho, tais indicadores informaram se a execução do plano pré-agendado sai como planejado. Isso impedirá uma interpretação incorreta dos resultados obtidos, ou seja, o sistema deve saber se o planejamento não foi bom ou não foi feito conforme planejamento. Portanto os indicadores principais para o processo de melhoria serão a estabilidade e robustez da solução preditiva, definidos na seção anterior.

\subsection{Fase de Ações de Melhoria}

Adotando a proposta de Gerolamo (2003), que propõem um processo sistematizado para gestão de melhorias e mudanças de desempenho, o macroprocesso de melhoria segue uma sequência de atividades inseridas no ciclo PDCA. O processo de melhoria contínua será dado através de ciclos de acompanhamento com período igual ao horizonte curto de planejamento. Em cada fim de ciclo os indicadores serão calculados e as adaptações sugeridas pelo método serão implementadas para a programação do próximo período.

Categorizando as atividades dentro do ciclo têm-se as definições dos parâmetros da programação na etapa de planejamento $(\mathrm{P})$. A definição dos novos parâmetros do sequenciamento de operações como tempos, buffers e escolha das técnicas é tratada na etapa de execução (D), então após o período do horizonte curto, os resultados são avaliados através dos indicadores de desempenho (C) e podem ser definitivos ou não de acordo com a evolução de desempenho mostrado (A). Então novas ações podem ser propostas e inicia-se outro ciclo.

\subsection{Arquitetura e Interface entre Sistemas}

Como descrito por Liu et al. (2002) a utilização deste método requer a implantação das várias ferramentas já mencionadas, para a proposta deste trabalho se tem que a integração entre os sistemas pode ser descrita conforme diagrama de colaboração apresentada na Figura 2.

Semelhante ao diagrama apresentado pelo método MAP-Prod os sistemas desempenham diferentes atividades no processo, por isso as informações devem ser compartilhadas pelas ferramentas para permitir a execução do método de forma correta e alinhada com todos os envolvidos.

\subsection{Considerações do Métodos MAP-Prod}

Percebe-se no método MAP-Prod a adaptabilidade do processo de programação da produção através da combinação de características das abordagens proativa e reativa, que 


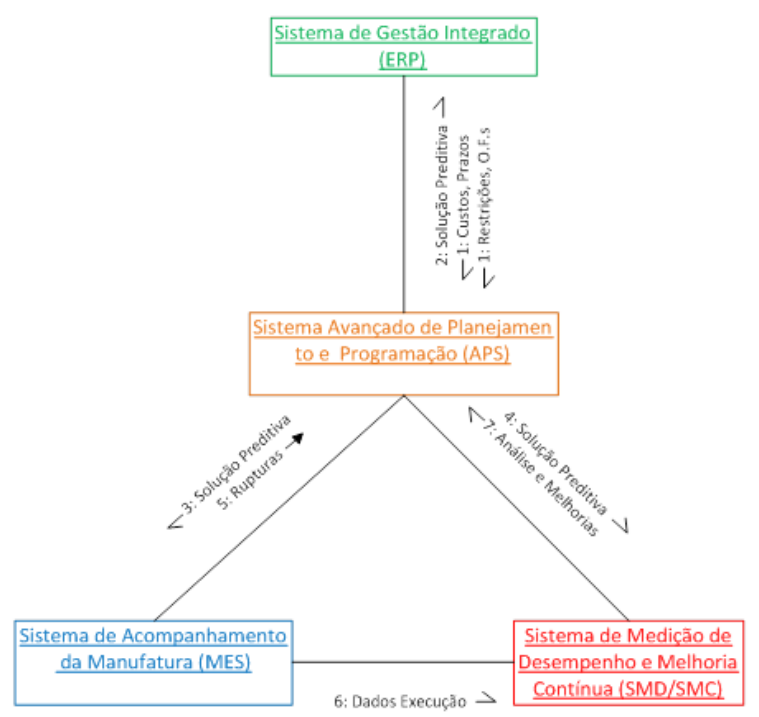

Figure 2. Integração entre os sistemas cooperantes para a proposta de sistema adaptativo

juntas proporcionam um processo de adequação momentânea aos desvios do planejamento e em longo prazo através do processo de melhoria realizado entre os ciclos de planejamento.

A capacidade de gerar soluções preditivas melhores e incorporar as ações de melhoria sugeridas durante a atualização de parâmetros está fortemente ligado a quantidade de parâmetros e técnicas de sequenciamento implementadas na ferramenta APS. O desempenho de cada técnica pode estar atrelado ao sistema de produção adotado por cada indústria e o sucesso de cada simulação pode depender a quantidade de cenários e objetivos que se possa adotar na ferramenta.

O MAP-Prod pode se tornar um método de difícil implantação devido a combinações de várias ferramentas e a necessidade de implementar rotinas para integração entre os sistemas. Mas é justamente a união das funcionalidades dos sistemas que torna o método um forte aliado na busca de um processo de fabricação eficiente. $\mathrm{O}$ processo de melhoria continua além de auxiliar no método de programação da produção pode gerar mudanças na cultura da empresa promovendo ações propostas pelos próprios funcionários.

Durante o processo de melhoria contínua pesquisado na literatura não houve nenhuma menção sobre a utilização de ferramentas para auxiliar e dar maior visibilidade do mesmo, por isso neste trabalho propôs-se a utilização de um sistema de medição de desempenho e melhoria contínua para gerenciar este processo de adaptação.

\section{Estudo de Caso: Aplicação do MAP-Prod na Indústria Moveleira}

Nesta seção serão demonstrados a aplicação da proposta, e os resultados que podem ser obtidos com a utilização do MAP-Prod através de um estudo de caso realizado em uma indústria do setor moveleiro. O foco será na análise do comportamento das ferramentas e o grau da adaptação dos parâmetros utilizados no sequenciamento de operação. Os indicadores de desempenho organizacional mostrarão o avanço da empresa quanto 


\begin{tabular}{|l|l|l|l|l|l|} 
SEMANA & $\begin{array}{l}N^{0} \text { de } \\
\text { O.P.s }\end{array}$ & $\begin{array}{l}\text { Peças } / \\
\text { Produtos }\end{array}$ & $\begin{array}{l}\text { Lead Time } \\
\text { Coletado }\end{array}$ & $\begin{array}{l}\text { Lead Time } \\
\text { APS }\end{array}$ & Diferença \\
\hline Semana 1 & 224 & 242.586 & $7: 15$ & $5: 27$ & $24,82 \%$ \\
\hline Semana 2 & 448 & 514.276 & $10: 10$ & $6: 29$ & $36,23 \%$ \\
\hline Semana 3 & 776 & 537.558 & $9: 16$ & $5: 44$ & $38,13 \%$ \\
\hline Semana 4 & 1122 & 1.098 .398 & $11: 23$ & $7: 22$ & $35,29 \%$
\end{tabular}

\section{Table 1. Resultados obtidos na simulação com a ferramenta APS e método adap- tativo MAP-Prod}

à programação da produção com a aplicação do método adaptativo.

Para a aplicação do método, é possível utilizar os sistemas existentes no mercado quando estes possam trabalhar de forma integrada conforme a arquitetura apresentada na Figura 2.As ferramentas utilizadas neste trabalho foram escolhidas pelo conhecimento adquirido pelo autor deste trabalho durante a participação nas equipes de desenvolvimento destas e por se tratar de sistemas que já apresentam alguma forma de integração conforme a arquitetura apresentada na Figura 2.

O problema foi formulado com as ordens de produção geradas durante um mês em horizontes semanais de planejamento elaborando-se três simulações, sendo a primeira uma simulação da aplicação das técnicas de sequenciamento através da ferramenta APS sobre as ordens de produção para a verificação de uma otimização do lead time dos produtos. Para a segunda procurou-se verificar a simulação dos parâmetros do método de programação às reais condições dos recursos fabris da empresa, para tal optou-se uma comparação da evolução dos tempos de processamento padrões conforme a análise semanal da produção para os tempos executados no horizonte seguinte.

Já na terceira simulação verificaram-se os diferenciais na utilização do Sistema de Medição de Desempenho e Melhoria Contínua como ferramenta de apoio e gerenciamento deste processo de melhoria do método de programação através da visualização de indicadores e acompanhamento do ciclo PDCA das ações de melhoria geradas durante a adaptação dos parâmetros da programação da produção.

\subsection{Resultados}

Como pode ser visto na Tabela 1, a produção durante as quatro semanas variaram bastante, pelo menos se analisarmos somente os números de peças e ordens de produção, porém como os produtos do setor moveleiro são de diferentes tamanhos e complexidade essa diferença pode ser justificada. Contudo a otimização do lead time dos produtos é confirmada em todas as semanas de produção, fator esse já esperado devido a enorme quantidade de material parado no chão de fábrica da empresa e do método de programação aplicado para a execução das ordens. O ganho médio das quatro semanas ficou em torno de $36,62 \%$, o que pode indicar fortemente um benefício na utilização da ferramenta APS pela empresa no que tange o estoque em processo e liquidez dos investimentos.

Para averiguar e mensurar a adaptação dos tempos para a geração das soluções preditivas utilizou-se os indicadores de estabilidade e robustez. Nos resultados pode-se verificar a aproximação dos valores referentes aos tempos padrões ajustados com os tem- 

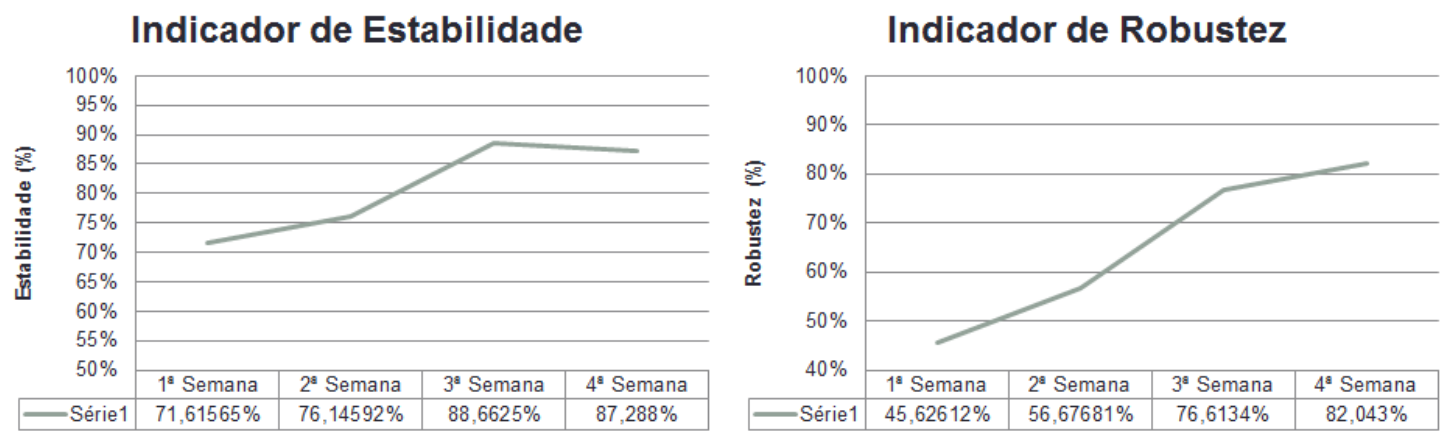

Figure 3. Indicadores de Estabilidade e Robustes para a simulação

pos praticados nas semanas seguintes a adaptação, indicação mostrada através da ascensão do indicador de estabilidade que passou de $71,61 \%$ na primeira semana para $87,28 \%$ na quarta semana (Gráfico 1 da Figura 3). Para o indicador de robustez tem-se uma melhora constante durante o mês de análise saindo de um valor de 45,62\% na primeira semana para 82,04\% ao final do mês (Gráfico 2 da Figura 3).

A evolução dos indicadores provavelmente deve tender para uma situação estável quando os parâmetros atingirem valores próximos aos ideais para utilização da ferramenta APS, provavelmente esta aproximação poderia ser verificada em um período maior de experimentação.

Verificou-se também a inserção das ferramentas de medição de desempenho e melhoria contínua no processo adaptativo de programação da produção, para isso parametrizou-se as ferramentas através do modelo BSC (Balanced Scorecard) proposto pela ferramenta escolhida com o cadastro de diferentes perspectivas e objetivos da empresa.

A ferramenta se demonstrou útil para a visualização dos resultados e análise dos dados coletados, além de gerenciar de forma colaborativa (alocação de responsáveis e acesso restrito por perfil de acesso) a coleta de indicadores quantitativos e qualitativos através de digitação manual ou importação de arquivos.Para facilitar a visualização dos valores, a ferramenta utiliza-se de gráficos e uso de cores indicativas de desempenho que são calculadas através do cadastro de limites inferiores e superiores e metas por período.

\section{Considerações Finais}

A integração entre as ferramentas presentes no método MAP-Prod pode ser realizada com o intuito de viabilizá-lo, além de trazer inúmeros benefícios para a análise de informações da empresa. Porém a maioria das ferramentas disponíveis no mercado necessita de esforços adicionais para sua comunicação com as demais, já que envolvem muitas vezes a comunicação entre sistemas de diferentes tecnologias e diferentes direitos autorais, tornando-a assim uma tarefa complexa para as empresas que a adquirem.

O uso das técnicas de sequenciamento através de sistemas APS e o método MAPProd se mostraram promissores através da análise do estudo de caso com resultados positivos para a otimização dos recursos e aumento da estabilidade e robustez das soluções. O método adaptativo proposto pelo presente trabalho pode solucionar o problema da dificuldade de parametrização dos métodos aplicando um ciclo de adaptações nos dados de 
entrada para o sistema de programação da produção condicionando as técnicas de sequenciamento a gerar sempre soluções mais compatíveis com a situação produtiva da empresa.

Outra grande vantagem do método adaptativo são as frequentes correções realizadas para adequar o método as mudanças normais de um processo fabril como: produção de novos produtos; aquisição ou troca de equipamentos; rotatividade de funcionários; e mudanças no processo de fabricação.

Metodologias como a medição de desempenho e melhoria contínua são essenciais para acompanhar esse processo evolutivo, garantindo o gerenciamento do método. Além de trazer inúmeros benefícios inerentes às metodologias.

\section{References}

Attadia, L. C. d. L. and Martins, R. A. (2003). Medição de desempenho como base para evolução da melhoria contínua. Prod, 13(2):33-41.

Aytug, H., Lawley, M. A., McKay, K., Mohan, S., and Uzsoy, R. (2005). Executing production schedules in the face of uncertainties: A review and some future directions. European Journal Of Operational Research, pages 86-110.

Bessant, J., Caffyn, S., Gilbert, J., R., H., and Webb, S. (1994). Rediscovering continous improvement. Technovation, 14.

Bidot, J., Vidal, T., Laborie, P., and Beck, J. C. (2009). A theoretic and practical framework for scheduling in a stochastic environment. Journal of Scheduling, 10.

Billaut, J. C., Moukrim, A., and Sanlaville, E. (2010). Introduction to Flexibility and Robustness in Scheduling. ISTE.

Gerolamo, M. C. Proposta de sistematização para o processo de gestão de melhorias e mudanças de desempenho. Master's thesis, São Carlos.

Herroelen, W. and Leus, R. (2005). Project scheduling under uncertainty: Survey and research potentials. European Journal Of Operational Research, pages 289-306.

Lambrechts, O., Demeulemeester, E., and Herroelen, W. (2008). Proactive and reactive strategies for resource-constrained project scheduling with uncertain resource availabilities. Journal of Scheduling, 11.

Liu, W., Chua, T. J., Lam, J., Wang, F. Y., Cai, T. X., and Yin, X. F. (2002). Aps, erp and mes systems integration for semiconductor backend assembly. 7th International Conference on Control, Automation, Robotic and Vision (ICARCV 2002), pages 14031408.

Mehta, S. and Uzsoy, R. (1998). Predictable scheduling of a job shop subject to breakdowns. IEEE Transactions on Robotics and Automation, 14:365-378.

Merli, G. (1993). EuroChallenge: TQM Approach to Capturing Global Markets. IFS.

Ouelhadj, D. and Petrovic, S. (2009). Survey of dynamic scheduling in manufacturing systems. Journal of Scheduling, 12/4:417-431.

Savolainen, T. (1999). Cycles of continuous improvement: realizing competitive advantages through quality. International Journal of Operations \& Production Management, 19(11). 